JOURNAL OF THE

AMERICAN MATHEMATICAL SOCIETY

Volume 21, Number 3, July 2008, Pages 775-795

S 0894-0347(08)00592-4

Article electronically published on March 18, 2008

\title{
ON THE RADIUS OF INJECTIVITY OF NULL HYPERSURFACES
}

\author{
SERGIU KLAINERMAN AND IGOR RODNIANSKI
}

\section{INTRODUCTION}

This paper is concerned with the regularity properties of boundaries $\mathcal{N}^{ \pm}(p)=$ $\partial \mathcal{I}^{ \pm}(p)$ of the past (future) sets of points in a $3+1$ Lorentzian manifold $(\mathbf{M}, \mathbf{g})$. The past of a point $p$, denoted $\mathcal{I}^{-}(p)$, is the collection of points that can be reached by a past directed time-like curve from $p$. As it is well known the boundaries $\mathcal{N}^{\mp}(p)$ play a crucial role in understanding the causal structure of Lorentzian manifolds and the propagation properties of linear and nonlinear waves, e.g. in flat spacetime the null cone $\mathcal{N}^{-}(p) \cup \mathcal{N}^{+}(p)$ is exactly the propagation set of solutions to the standard wave equation with a Dirac measure source point at $p$. However these past (future) boundaries fail, in general, to be smooth even in a smooth, curved, Lorentzian space-time; one can only guarantee that $\mathcal{N}^{ \pm}(p)$ is a Lipschitz, achronal, 3 -dimensional manifold without boundary ruled by in-extendible null geodesics from $p$; see [HE]. In fact $\mathcal{N}^{ \pm}(p) \backslash\{p\}$ is smooth in a small neighborhood of $p$ but fails to be so in the large because of conjugate points, resulting in formation of caustics, or because of intersections of distinct null geodesics from $p$. Providing a lower bound for the radius of injectivity of the sets $\mathcal{N}^{ \pm}(p)$ is thus an essential step in understanding more refined properties of solutions to linear and nonlinear wave equations in a Lorentzian background.

The phenomenon described above is also present in Riemannian geometry in the context of geodesic coordinates relative to a point, yet in that case the presence of conjugate or cut-locus points has nothing to do with the regularity of the manifold itself. In that sense lower bounds for the radius of injectivity of a Riemannian manifold are important only insofar as geodesic normal coordinates, and their applications 11 are concerned. Thus, for example, lower bounds for the radius of injectivity can sometimes be replaced by lower bounds for the harmonic radius, which plays an important role in Cheeger-Gromov theory; see e.g. A2.

In this paper we investigate regularity of past (future) boundaries $\mathcal{N}^{\mp}(p)$ in Einstein vacuum space-times, i.e. Lorentzian manifolds $(\mathbf{M}, \mathbf{g})$ with the Ricci flat metric, $\mathbf{R}_{\alpha \beta}(\mathbf{g})=0$. We provide conditions 2 expressed in terms of a space-like foliation $\Sigma_{t}$, given by the level surfaces of a regular time function $t$ with unit future

Received by the editors March 5, 2006.

2000 Mathematics Subject Classification. Primary 35J10.

The first author is supported by NSF grant DMS-0070696.

The second author is partially supported by NSF grant DMS-0406627.

${ }^{1}$ Such as, for example, Sobolev inequalities or the finiteness theorem of Cheeger.

${ }^{2}$ These conditions imply, in particular, uniform bounds for the curvature flux, i.e. $L^{2}$-integrals along these boundaries for the tangential components of the curvature tensor

(C) 2008 American Mathematical Society Reverts to public domain 28 years from publication 
normal $\mathbf{T}$ sufficient to ensure the local nondegeneracy of these boundaries. More precisely we provide a uniform lower bound on the radius of injectivity of the null boundaries $\mathcal{N}^{ \pm}(p)$ of the causal past (future) sets $\mathcal{J}^{ \pm}(p)$. Such lower bounds are essential in understanding the causal structure and the related propagation properties of solutions to the Einstein equations. They are particularly important in the construction of an effective Kirchoff-Sobolev type parametrix for solutions of wave equations on $\mathbf{M}$; see $\mathrm{KR} 4$. These parametrices are used in KR5, to prove a large data break-down criterion for solutions of the Einstein-vacuum equations.

This work complements our series of papers [KR1- KR3]. The methods developed in those papers can be adapted ${ }^{3}$ to prove lower bounds on the radius of conjugacy with respect to the parameter 4 of the $\Sigma_{t}$ foliation. It may, however, be possible that the radius of conjugacy of the null congruence is bounded from below, and yet there are past null geodesics that form a point $p$ intersecting again at points arbitrarily close to $p$. Indeed, this does happen on a flat Lorentzian manifold $\mathbf{M}=\mathbb{T}^{3} \times \mathbb{R}$ where $\mathbb{T}^{3}$ is the torus obtained by identifying the opposite sides of a lattice of period $L$ and the metric induced by the standard Minkowski metric. Clearly there can be no conjugate points for the congruence of past or future null geodesics from a point, and yet there are plenty of distinct null geodesics from a point $p$ in $\mathbf{M}$ which intersect on a time scale proportional to $L$. Thus there can be no lower bounds on the null radius of injectivity expressed only in terms of bounds for the curvature tensor R. This problem also occurs, of course, in Riemannian geometry, where we can control the radius of conjugacy in terms of uniform bounds for the curvature tensor, yet, in order to control the radius of injectivity we need to make other geometric assumptions such as, in the case of compact Riemannian manifolds, lower bounds on volume and upper bounds for its diameter. Thus it should come as no surprise that we also need, in addition to conditions which guarantee bounds for the curvature flux, other assumptions on the geometry of solutions to the Einstein equations in order to ensure control on the null cut-locus of points in $\mathbf{M}$ and obtain lower bounds for the null radius of injectivity.

We discuss two related results. In the first result we also assume (in addition to the conditions which ensure the boundedness of the curvature flux, using energy type estimates for the Bel-Robinson tensor; see section 5) that every point of the space-time admits a sufficiently large coordinate patch with a system of coordinates in which the Lorentz metric $\mathbf{g}$ is close to a flat Minkowski metric. In the second result we dispense of the latter condition by showing how such coordinates can be constructed, dynamically, from a given coordinate system on the initial slice $\Sigma_{0}$. Though the second result (Main Theorem II) is more appropriate for applications, the main new ideas of the paper appear in section 2 related to the proof of the first result (Main Theorem I).

The uniform bounds on the curvature flux mentioned above (derived by energy estimates in section 5) are enough, according to the results in [KR1-[KR3] and

\footnotetext{
${ }^{3}$ In [KR1]-[KR3] we have considered the case of the congruence of outgoing future null geodesics initiating on a 2 -surface $S_{0}$ embedded in a space-like hypersurface $\Sigma_{0}$. The extension of our results to null cones from a point forms the subject of Qian Wang's Princeton 2006 Ph.D. thesis; see Wang.

${ }^{4}$ The results in KR1- KR3] and Wang were proved with respect to the geodesic foliation. In this paper we assume that the results can be extended to the foliation on $\mathcal{N}^{ \pm}(p)$ induced by the space-like foliation $\Sigma_{t}$. The formal proof will be given elsewhere. For further discussion see the comments after Theorem 2.13
} 
Wang, to give us control on the radius of conjugacy of the corresponding null congruence. There is, however, an important subtlety involved here. Past (future) points of intersection, distinct null geodesics from $p$ are no longer on the boundary of $\mathcal{J}^{\mp}(p)$ and therefore the energy estimates mentioned above do not apply. Consequently we cannot simply apply the results [KR1- KR3] and estimate the null radius of conjugacy independent of the cut-locus, but we have to treat them together by a delicate boot-strap argument. The main new ideas of this paper concern estimates for the cut locus, i.e. establishing lower bounds, with respect to the time parameter $t$, for the points of intersection of distinct past (future) null geodesics from $p$. Though our results, as formulated, hold only for Einstein vacuum manifolds, our method of proof in section 2 can be extended to general Lorentzian manifolds if we make, in addition to the assumptions mentioned above, uniform norm assumptions for the curvature tensor $\mathbf{R}$. Our results seem to be new, even in this vastly simplified case; indeed we are not aware of any nontrivial results concerning the null radius of injectivity for Lorentzian manifolds.

It is instructive to make a comparison with the corresponding picture in Riemannian geometry. In general, all known lower bounds on the radius of injectivity require some pointwise control of the curvature. The gold standard in this regard is a theorem of Cheeger providing a lower bound on the radius of injectivity in terms of pointwise bounds on the sectional curvature and diameter, and a lower bound on the volume of a compact manifold; see [Ch. As in our case, the Riemannian geometry problem can be handled by splitting it into a lower bound on the radius of conjugacy and a separate estimate on the length of the shortest geodesic loop. The radius of conjugacy is intimately tied to pointwise bounds on curvature via the Jacobi equation. The estimate for the length of the shortest geodesic loop, on the other hand, relied, traditionally, on Toponogov's Theorem, which again needs pointwise bounds on the curvature. These two problems can be naturally separated in Riemannian geometry 5 and while the radius of conjugacy requires pointwise assumptions on curvature, a lower bound on the length of the shortest geodesic can be given under weaker, integral, assumptions on curvature. The best result in the latter direction, to our knowledge, is due to Petersen-Steingold-Wei, which in addition to the usual diameter and volume conditions on an $n$-dimensional compact manifold, requires smallness of the $L^{p}$-norm of sectional curvature, for $p>n-1$ [PSW]. Once more, we want to re-emphasize the fact that in Riemannian geometry lower bounds on the radius of injectivity require pointwise bounds for the curvature, yet this restriction can often be overcome in applications by replacing it with bounds for more flexible geometric quantities. A case in point is the Anderson-Cheeger result $[\mathrm{AC}]$ which proves a finiteness theorem under pointwise assumptions on the Ricci curvature and $L^{\frac{n}{2}}$ bounds on the full Riemann curvature tensor. Unlike the classical result of Cheeger, see $\mathrm{Ch}$, the radius of injectivity need not, and cannot in general, be estimated.

The works in Riemannian geometry, cited above, have been largely stimulated by applications to the Cheeger-Gromov theory. Applications of this theory to general relativity have been pioneered by M. Anderson, A2 and A3]; see also [KR5] for further applications. M. Anderson has, in particular, been interested in the possibility of adapting some aspects of Cheeger-Gromov theory to the Lorentzian

\footnotetext{
${ }^{5}$ Something that we do not know how to do in the Lorentzian case.
} 
setting. With this in mind he has proved the existence of a special space-time coordinate system for Einstein vacuum space-times, under pointwise assumptions on the space-time Riemann curvature tensor; see A1. Another example of a well known, global, geometric result which has been successfully adapted from the Riemannian to Lorentzian setting is Galloway's null splitting theorem; see [G].

Finally we note that, in the Riemannian setting, the radius of injectivity and shortest geodesic loop estimates depend crucially on lower bounds for the volume of the manifold, as confirmed by the example of a thin flat torus. By contrast, the notion of the volume of a null hypersurface in Lorentzian geometry is not well-defined, as the restriction of the space-time metric to a null hypersurface is degenerate. We are forced to replace the condition on the volume of $\mathcal{N}^{\mp}(p)$ with the condition on the volume of the 3 -dimensional domain obtained by intersecting the causal past (future) of $p$ with the level hypersurfaces of a time function $t$. To be more precise, in the case of $\mathcal{N}^{-}(p)$, our assumption on the existence of a coordinate system in which the metric $\mathbf{g}$ is close to the Minkowski metric will allow us to prove that the volume of these domains, at time $t<t(p)$, are close to the volume of the Euclidean ball of radius $t(p)-t$, where $t(p)$ denotes the value of the parameter $t$ at $p$. Another ingredient of our proof is an argument showing that, at the first time of intersection $q$, past null geodesics from a point $p$ meet each other at angle $\pi$, viewed with respect to the tangent space of the space-like hypersurface $t=t(q)$. We also show that we can find a point $p$, such that the above property holds both at $p$ and the first intersection point $q$. Finally, we give a geometric comparison argument showing that the existence of a pair of null geodesics from a point $p$ meeting each other at angle $\pi$ both at $p$ and at the point of first intersection violates the structure of the past set $\mathcal{J}^{-}(p)$, if the time of intersection is too close to the value $t(p)$.

\section{MAin Results}

We consider a space-time $(\mathbf{M}, \mathbf{g})$ verifying the Einstein-vacuum equations,

$$
\mathbf{R}_{\alpha \beta}=0,
$$

and assume that a part of the space-time $\mathcal{M}_{I} \subset \mathbf{M}$ is foliated by the level hypersurfaces of a time function $t$, monotonically increasing towards the future in the interval $I \subset \mathbb{R}$. We shall also assume that $\mathcal{M}_{I}$ is globally hyperbolic. Without loss of generality we shall assume further that the length of $I$ verifies

$$
|I| \geq 1 \text {. }
$$

Let $\Sigma_{0}$ be a fixed leaf of the $t$ foliation. Starting with a local coordinates chart $U \subset \Sigma_{0}$ and coordinates $\left(x^{1}, x^{2}, x^{3}\right)$ we parametrize the domain $I \times U \subset \mathcal{M}_{I}$ with transported coordinates $\left(t, x^{1}, x^{2}, x^{3}\right)$ obtained by following the integral curves of $\mathbf{T}$, the future unit normal to $\Sigma_{t}$. The space-time metric $\mathbf{g}$ on $I \times U$ then takes the form

$$
\mathbf{g}=-n^{2} d t^{2}+g_{i j} d x^{i} d x^{j}
$$

where $n$ is the lapse function of the $t$ foliation and $g$ is the restriction of the metric $\mathrm{g}$ to the surfaces $\Sigma_{t}$ of constant $t$. We shall assume that the space-time region $\mathcal{M}_{I}$ is globally hyperbolic, i.e. every causal curve from a point $p \in \mathcal{M}_{I}$ intersects $\Sigma_{0}$ at precisely one point.

The second fundamental form of $\Sigma_{t}$ is defined by

$$
k(X, Y)=\mathbf{g}\left(\mathbf{D}_{X} \mathbf{T}, Y\right), \quad \forall X, Y \in T\left(\Sigma_{t}\right),
$$


with $\mathbf{D}$ the Levi-Civita covariant derivative. Observe that

$$
\partial_{t} g_{i j}=-2 n k_{i j}
$$

We denote by $\operatorname{tr} k$ the trace of $k$ relative to $g$, i.e. $\operatorname{tr} k=g^{i j} k_{i j}$. We also assume that the surfaces $\Sigma_{t}$ are either compact or asymptotically flat.

Given a unit time-like normal $\mathbf{T}$ we can define a pointwise norm $|\Pi(p)|$ of any space-time tensor $\Pi$ with the help of the decomposition

$$
X=X^{0} \mathbf{T}+\underline{X}, \quad X \in T \mathcal{M}, \quad \underline{X} \in T \Sigma_{t} .
$$

The norm $|\Pi(p)|$ is then defined relative to the Riemannian metric,

$$
\overline{\mathbf{g}}(X, Y)=X^{0} \cdot Y^{0}+g(\underline{X}, \underline{Y}) .
$$

We denote by $\|\Pi(t)\|_{L^{p}}$ the $L^{p}$ norm of $\Pi$ on $\Sigma_{t}$. More precisely,

$$
\|\Pi(t)\|_{L^{p}}=\int_{\Sigma_{t}}|\Pi|^{p} d v_{g}
$$

with $d v_{g}$ the volume element of the metric $g$ of $\Sigma_{t}$.

Let ${ }^{(\mathbf{T})} \pi=\mathcal{L}_{\mathbf{T}} \mathbf{g}$ be the deformation tensor of $\mathbf{T}$. The components of ${ }^{(\mathbf{T})} \pi$ are given by

$$
{ }^{(\mathbf{T})} \pi_{00}=0, \quad{ }^{(\mathbf{T})} \pi_{0 i}=\nabla_{i} \log n, \quad{ }^{(\mathbf{T})} \pi_{i j}=-2 k_{i j} .
$$

2.1. Main assumptions. We make the space-time assumption

$$
\begin{aligned}
N_{0}^{-1} & \leq n \leq N_{0}, \\
|I| \cdot \sup _{t \in I}\|\pi(t)\|_{L^{\infty}} & \leq \mathcal{K}_{0}<\infty,
\end{aligned}
$$

where $N_{0}, \mathcal{K}_{0}>0$ are given numbers and $|I|$ denotes the length of the time interval $I \subset \mathbb{R}$. We also make the following assumptions on the initial hypersurface $\Sigma_{0}$.

I1. There exists a covering of $\Sigma_{0}$ by charts $U$ such that for any fixed chart, the induced metric $g$ verifies

$$
I_{0}^{-1}|\xi|^{2} \leq g_{i j}(x) \xi_{i} \xi_{j} \leq I_{0}|\xi|^{2}, \quad \forall x \in U
$$

with $I_{0}$ a fixed positive number. Moreover there exists a number $\rho_{0}>0$ such that every point $y \in \Sigma_{0}$ admits a neighborhood $B$, included in a neighborhood chart $U$, such that $B$ is precisely the Euclidean ball $B=B_{\rho_{0}}^{(e)}(y)$ relative to the local coordinates in $U$.

I2. The Ricci curvature of the initial slice $\Sigma_{0}$ verifies

$$
\|\mathbf{R}\|_{L^{2}\left(\Sigma_{0}\right)} \leq \mathcal{R}_{0}<\infty .
$$

Remark. If $\Sigma_{0}$ is compact the existence of $\rho_{0}>0$ is guaranteed by the existence of the coordinates charts $U$ verifying (17). More precisely we have:

Lemma 2.2. If $\Sigma_{0}$ is compact and has a system of coordinate charts $U$ verifying (77), there must exist a number $\rho_{0}>0$ such that every point $y \in \Sigma_{0}$ admits a neighborhood $B$, included in a neighborhood chart $U$, such that $B$ is precisely the Euclidean ball $B=B_{\rho_{0}}^{(e)}(y)$ relative to the local coordinates in $U$.

Proof. According to our assumption every point $x \in \Sigma_{0}$ belongs to a coordinate patch $U$. Let $r(x)>0$ be such that the euclidean ball, with respect to the coordinates of $U$, centered at $x$ of radius $r(x)$, is included in $U$. Due to compactness of $\Sigma_{0}$ we can find a finite number of points $x_{1}, \ldots, x_{N}$ such that the balls $B_{r_{j} / 2}^{(e)}\left(x_{j}\right)$, with $r_{j}=r\left(x_{j}\right)$ for $j=1, \ldots, N$, cover $\Sigma_{0}$. Thus any $y \in \Sigma_{0}$ must belong to a ball 
$B_{r_{j} / 2}^{(e)}\left(x_{j}\right) \subset B_{r_{j}}^{(e)}\left(x_{j}\right) \subset U$, for some $U$. Therefore the ball $B_{r_{j} / 2}^{(e)}(y) \subset U$. We then choose $\rho_{0}=\min _{j=1}^{N} r_{j} / 2$.

2.3. Null boundaries. Starting with any point $p \in \mathcal{M}_{I} \subset \mathbf{M} 6$ we denote by $\mathcal{J}^{-}(p)=\mathcal{J}^{-}\left(p ; \mathcal{M}_{I}\right)$ the causal past of $p$, relative to $\mathcal{M}_{I}$, by $\mathcal{I}^{-}(p)$ its interior and by $\mathcal{N}^{-}(p)$ its null boundary. In the same vein 7 reversing the direction of time, we define $\mathcal{J}^{+}(p)=\mathcal{J}^{+}\left(p ; \mathcal{M}_{I}\right), \mathcal{I}^{+}(p)$ and $\mathcal{N}^{+}(p)$. In general $\mathcal{N}^{-}(p)$ is an achronal, Lipschitz hypersurface, ruled by the set of past null geodesics from $p$. We parametrize these geodesics with respect to the future, unit, time-like vector $\mathbf{T}_{p}$. Then, for every direction $\omega \in \mathbb{S}^{2}$, with $\mathbb{S}^{2}$ denoting the standard sphere in $\mathbb{R}^{3}$, consider the null vector $\ell_{\omega}$ in $T_{p} \mathbf{M}$,

$$
\mathbf{g}\left(\ell_{\omega}, \mathbf{T}_{p}\right)=1
$$

and associate to it the past null geodesic $\gamma_{\omega}(s)$ with initial data $\gamma_{\omega}(0)=p$ and $\dot{\gamma}_{\omega}(0)=\ell_{\omega}$. We further define a null vectorfield $\mathbf{L}$ on $\mathcal{N}^{-}(p)$ according to

$$
\mathbf{L}\left(\gamma_{\omega}(s)\right)=\dot{\gamma}_{\omega}(s)
$$

where $\mathbf{L}$ may only be smooth almost everywhere on $\mathcal{N}^{-}(p)$ and can be multi-valued on a set of exceptional points. We can choose the parameter $s$ in such a way that $\mathbf{L}=\dot{\gamma}_{\omega}(s)$ is geodesic and $\mathbf{L}(s)=1$.

For a sufficiently small $\delta>0$ the exponential map $\mathcal{G}=\mathcal{G}_{p}^{-}$, defined by

$$
(s, \omega) \rightarrow \gamma_{\omega}(s),
$$

is a diffeomorphism from $(0, \delta) \times \mathbb{S}^{2}$ to its image in $\mathcal{N}^{-}(p)$. Moreover for each $\omega \in \mathbb{S}^{2}$ either $\gamma_{\omega}(s)$ can be continued for all positive values of $s$ or there exists a value $s_{*}(\omega)$ beyond which the points $\gamma_{\omega}(s)$ are no longer on the boundary $\mathcal{N}^{-}(p)$ of $\mathcal{J}^{-}(p)$ but rather in its interior; see $\mathrm{HE}$. We call such points terminal points of $\mathcal{N}^{-}(p)$. We say that a terminal point $q=\gamma_{\omega}\left(s_{*}\right)$ is a conjugate terminal point if the map $\mathcal{G}$ is singular at $\left(s_{*}, \omega\right)$. A terminal point $q=\gamma_{\omega}\left(s_{*}\right)$ is said to be a cut locus terminal point if the map $\mathcal{G}=\mathcal{G}_{p}^{-}$is nonsingular at $\left(s_{*}, \omega\right)$ and there exists another null geodesic from $p$, passing through $q$.

Thus $\mathcal{N}^{-}(p)$ is a smooth manifold at all points except the vertex $p$ and the terminal points of its past null geodesic generators. We denote by $\mathcal{T}^{-}(p)$ the set of all terminal points and by $\dot{\mathcal{N}}^{-}(p)=\mathcal{N}^{-}(p) \backslash \mathcal{T}^{-}(p)$ the smooth portion of $\mathcal{N}^{-}(p)$. The set $\mathcal{G}^{-1}\left(\mathcal{T}^{-}(p)\right)$ has measure zero relative to the standard measure $d s d a_{\mathbb{S}^{2}}$ of the cone $[0, \infty) \times \mathbb{S}^{2}$. We will denote by $d A_{\mathcal{N}^{-}(p)}$ the corresponding measure on $\mathcal{N}^{-}(p)$. Observe that the definition is not intrinsic; it depends in fact on the normalization condition (9).

Definition 2.4. We define $i_{*}^{-}(p)$ to be the supremum over all the values $s>0$ for which the exponential map $\mathcal{G}_{p}^{-}:(s, \omega) \rightarrow \gamma_{\omega}(s)$ is a global diffeomorphism. We shall refer to $i_{*}^{-}(p)$ as the past null radius of injectivity at $p$ relative to the geodesic foliation defined by (9). Changing the direction of time we define, in precisely the same manner, the future null radius of injectivity $i_{*}^{+}(p)$.

\footnotetext{
${ }^{6}$ Recall that $\mathcal{M}_{I}$ is assumed to be globally hyperbolic.

${ }^{7}$ In what follows we concentrate on past domains. All definitions and statements concerning past domains can easily be adapted to future domains as well.

${ }^{8}$ For which $\gamma_{\omega}(s)$ stays in $\mathcal{M}_{I}$.
} 
Definition 2.5. We denote by $\ell_{*}^{-}(p)$ the smallest value of $s$ for which there exist two distinct, past directed, null geodesics $\gamma_{\omega_{1}}(s), \gamma_{\omega_{2}}(s)$ from $p$ which intersect at a point for which the corresponding value (smallest for $\gamma_{\omega_{1}}$ and $\gamma_{\omega_{2}}$ ) of the affine parameter is $s=\ell_{*}^{-}(p)$. We call $\ell_{*}^{-}(p)$ the past cut-locus radius at $p$. By symmetry we also define $l_{*}^{+}(p)$ as the future cut-locus radius at $p$.

Definition 2.6. Let $s_{*}^{-}(p)$ denote the supremum over all values of $s>0$ such that the exponential map $\mathcal{G}_{p}^{-}$, defined by (10), is a local diffeomorphism on $[0, s) \times \mathbb{S}^{2}$. We shall refer to $s_{*}^{-}(p)$ as the past null radius of conjugacy at the point $p$. Changing the direction of time we also define $s_{*}^{+}(p)$ as the future null radius of conjugacy at $p$.

Clearly,

$$
i_{*}^{ \pm}(p)=\min \left(l_{*}^{ \pm}(p), s_{*}^{ \pm}(p)\right) .
$$

Remark 2.7. Unlike Riemannian geometry, where the radius of injectivity is defined with respect to the distance function, the definitions above are not intrinsic but depend on (91). Moreover the definitions were made with respect to the affine parameter $s$ whose definition depends on both the point $p$ and normalization (9). In applications it makes more sense to refer to the global time parameter $t$.

We are thus led to the following:

Definition 2.8. Given $p \in \mathcal{M}_{I}$ we define $i_{*}^{-}(p, t)$ to be the supremum over all the values $t(p)-t, t<t(p)$, for which the exponential map $\mathcal{G}=\mathcal{G}_{p, t}^{-}$,

$$
(t, \omega) \rightarrow \gamma_{\omega}(t)=\gamma_{\omega}(s(t))
$$

is a global diffeomorphism. We denote by $\ell_{*}^{+}(p, t)$ the supremum over all the values $t(p)-t, t<t(p)$, for which there exist two distinct null geodesics $\gamma_{\omega_{1}}, \gamma_{\omega_{2}}$ from $p$ which intersect at a point on $\Sigma_{t}$. Similarly, we let $s_{*}^{-}(p, t)$ be the supremum of $t(p)-t$ for which the exponential map $\mathcal{G}(t, \omega)$ is a local diffeomorphism. We shall refer to $i_{*}^{-}(p, t), l_{*}^{-}(p, t), s_{*}^{-}(p, t)$, respectively, as the past null radius of injectivity, respectively, past cut-locus radius and and past radius of conjugacy, at $p$ relative to the $t$-foliation. By reversing the direction of time we also define $i_{*}^{+}(p, t), l_{*}^{+}(p, t), s_{*}^{+}(p, t)$.

Clearly,

$$
i_{*}^{ \pm}(p, t)=\min \left(l_{*}^{ \pm}(p, t), s_{*}^{ \pm}(p, t)\right) .
$$

To keep track of the past and future boundaries of $\mathcal{M}_{I}$ in $\mathbf{M}$ we also introduce:

Definition 2.9. We define $d^{ \pm}(p, t)$ to be the distances, measured with respect to the time parameter $t$, from $p$ to the past and future boundaries of $\mathcal{M}_{I} \subset \mathbf{M}$. Also,

$$
d(p, t)=\min \left(d^{-}(p, t), d^{+}(p, t)\right) .
$$

Remark 2.10. It is convenient to adjust the definition of $\ell_{*}^{ \pm}(p, t)$ (respectively $\left.s_{*}^{ \pm}(p, t)\right)$ in such a way that, if $\ell_{*}^{ \pm}(p, t)=d^{ \pm}(p, t)$ (respectively $s_{*}^{ \pm}(p, t)=d^{ \pm}(p, t)$ ) and no two geodesics from $p$ intersect at the corresponding boundary of $\mathcal{M}_{I}$ (respectively there are no conjugate points at the boundary of $\left.\mathcal{M}_{I}\right)$, then $\ell_{*}^{ \pm}(p, t)=\infty$ (respectively $s_{*}^{ \pm}(p, t)=\infty$ ).

The first goal of this paper is to prove Theorem 2.11 below, concerning lower, uniform bounds for the radii of injectivity $i_{*}^{ \pm}(p)$ of points $p$ in the space-time region $\mathcal{M}_{I}$, under the following additional assumptions: 
Assumption C. Every point $p \in \mathcal{M}_{I}$ admits a coordinate neighborhood $I_{p} \times U_{p}$ in $\mathbf{M}$ such that $U_{p}$ contains a geodesic ball $B_{r_{0}}(p)$ and $I_{p}$ is an interval around $t(p)$ with the property

$$
\sup _{t \in I_{p}} \pm(t-t(p)) \geq \min \left(r_{0}, d^{ \pm}(p, t)\right) .
$$

We assume that on $I_{p} \times U_{p}$ there exists a system of transported coordinates (2) close to the flat Minkowski metric $-n(p)^{2} d t^{2}+\delta_{i j} d x^{i} d x^{j}$. More precisely,

$$
\begin{aligned}
& |n(t, x)-n(p)|<\epsilon, \\
& \left|g_{i j}(t, x)-\delta_{i j}\right|<\epsilon,
\end{aligned}
$$

where $n(p)$ denotes the value of the lapse $n$ at $p$.

Assumption D. Assume that $\mathcal{M}_{I}$ contains a future, $t$-compact se $\mathcal{D}^{+} \subset \mathcal{M}_{I}$ and a past $t$-compact set $\mathcal{D}^{-} \subset \mathcal{M}_{I}$ such that there exists a positive constant $\delta_{0}$ with the property that for any point $q$ in the complement of $\mathcal{D}^{+}$we have $\ell_{*}^{-}(q, t)>$ $\min \left(\delta_{0}, d^{-}(q, t)\right)$ and for any $q$ in the complement of $\mathcal{D}^{-}$we have $\ell_{*}^{+}(q, t)>$ $\min \left(\delta_{0}, d^{+}(q, t)\right)$.

Theorem 2.11 (Main Theorem I). Assume that $\mathcal{M}_{I}$ is globally hyperbolic and verifies the main assumptions (5), (6), (8) as well as Assumptions $\mathbf{C}, \mathbf{D}$ above. Then, for sufficiently small $\epsilon>0$, there exists a positive number $i_{*}>0$, depending only on $\delta_{0}, r_{0}, N_{0}, \mathcal{K}_{0}$ and $\mathcal{R}_{0}$, such that, for all $p \in \mathcal{M}_{I}$,

$$
i_{*}^{ \pm}(p, t)>\min \left(i_{*}, d^{ \pm}(p, t)\right) .
$$

Assumption $\mathbf{C}$ of Theorem 2.11 can in fact be eliminated according to the following.

Theorem 2.12 (Main Theorem II). Assume that $\mathcal{M}_{I}$ is globally hyperbolic and verifies the assumptions (5), (6) as well as the initial assumptions I1 and I2. Also, assume Assumption $\mathbf{D}$ is verified. There exists a positive number $i_{*}>0$, depending only on $I_{0}, \rho_{0}, \delta_{0}, N_{0}, \mathcal{K}_{0}$ and $\mathcal{R}_{0}$, such that, for all $p \in \mathcal{M}_{I}$,

$$
i_{*}^{ \pm}(p, t)>\min \left(i_{*}, d^{ \pm}(p, t)\right) .
$$

Remark. Observe that Assumption $\mathbf{D}$ of both theorems, concerning a lower bound for $l_{*}$ outside a sufficiently large future compact set, is superfluous on a manifold with compact initial slice $10 \Sigma_{0}$. Thus, for manifolds $\mathcal{M}_{I}=I \times \Sigma_{0}$ with $\Sigma_{0}$ compact, $i_{*}$ depends only on the constants $I_{0}, N_{0}, \mathcal{K}_{0}$ and $\mathcal{R}_{0}$.

The first key step in the proof of both theorems is a lower bound on the radii of conjugacy $s_{*}^{ \pm}(p, t)$.

Theorem 2.13. There exists a sufficiently small constant $\delta_{*}>0$ depending only on $\mathcal{K}_{0}$ and $\mathcal{R}_{0}$ such that, for any $p \in \mathcal{M}_{I}$ we have,

$$
s_{*}^{ \pm}(p, t)>\min \left(\ell_{*}^{ \pm}(p, t), \delta_{*}, d^{ \pm}(p, t)\right) .
$$

Theorem 2.13 is very similar to the corresponding result 11 concerning the radius of conjugacy $s_{*}^{ \pm}(p)$ with respect to the geodesic foliation; see [KR1]-KR3] 12 The

\footnotetext{
${ }^{9}$ This means that its intersection with the level sets $\Sigma_{t}$ are compact.

${ }^{10} \mathrm{~A}$ similar statement can be made if $\Sigma_{0}$ is asymptotically flat.

${ }^{11}$ I.e. one proves the lower bound $s_{*}^{ \pm}(p)>\min \left(\ell_{*}^{ \pm}(p), \delta_{*}, d^{ \pm}(p)\right)$.

${ }^{12}$ See also Q. Wang's thesis, Princeton University, 2006 Wang.
} 
main assumption of that result was a bound on the flux of curvature across $\mathcal{N}^{ \pm}(p)$. Such bounds can easily be derived by energy estimates using the Bel-Robinson tensor and the bounds (5) and (6) on the lapse $n$ and second fundamental form $k$; see the derivation in section 5. A complete proof of Theorem 2.13, which is a rather straightforward adaptation of the proof in KR1]- KR3] as well as Wang], will appear elsewhere.

The results leading up to the proof of Theorem 2.13 also imply the following statement concerning the 2-surfaces of intersection between $\Sigma_{t}$ and the null boundaries $\mathcal{N}^{ \pm}(p)$.

Theorem 2.14. In addition to the results of Theorem 2.13 , under the same assumption, if

$$
0 \leq t(p)-t<\min \left(\ell_{*}^{-}(p, t), \delta_{*}, d^{-}(p, t)\right),
$$

the foliation $S_{t}=\Sigma_{t} \cap \mathcal{N}^{-}(p)$ is smooth. For these values of the metrics $\sigma_{t}$ on $\mathbb{S}^{2}$, obtained by restricting the metric $g_{t}$ on $\Sigma_{t}$ to $S_{t}$ and then pulling it back to $\mathbb{S}^{2}$ by the exponential map $\mathcal{G}(t, \cdot)$, we have

$$
\left|\sigma_{t}(X, X)-\sigma_{0}(X, X)\right| \leq \epsilon \sigma_{t}(X, X), \quad \forall X \in T \mathbb{S}^{2},
$$

where $\sigma_{0}$ is the standard metric on $\mathbb{S}^{2}$ and $\epsilon>0$ is a sufficiently small constant dependent on $\delta_{*}$.

A similar statement holds for the foliation induced by $\mathcal{N}^{+}(p)$.

In this paper we assume the conclusions of Theorem 2.14 and proceed with the proof of Main Theorem I.

\section{Proof of Main Theorem I}

According to Theorem 2.14 the desired conclusion of Main Theorem I will follow after finding a small constant $\delta_{*}$ dependent only on $\delta_{0}, r_{0}, N_{0}, \mathcal{R}_{0}$ and $\mathcal{K}_{0}$ with the property that $\ell_{*}^{ \pm}(p, t)>\min \left(\delta_{*}, d^{ \pm}(p, t)\right)$.

In what follows we consider the quantity $l_{*}^{-}(p, t)$; the arguments for $l_{*}^{+}(p, t)$ are exactly the same.

We fix $\delta_{*}$, to be chosen later, and assume that $\ell_{*}^{-}(p, t) \leq \min \left(\delta_{*}, d^{-}(p, t)\right)$. According to the convention introduced in Remark 2.10, if $d^{-}(p, t) \leq \delta_{*}$ and $\ell_{*}^{-}(p, t)=d^{-}(p, t)$ we can find two past geodesics from $p$ which intersect precisely at the past boundary of $\mathcal{M}_{I}$.

Recall that $g_{t}$ and $\sigma_{t}$ denote the restrictions of the space-time metric $\mathbf{g}$ to respectively $\Sigma_{t}$ and $S_{t}$, while $\sigma_{0}$ is the push-forward of the standard metric on $\mathbb{S}^{2}$ by the exponential map $\mathcal{G}^{-}(t, \cdot)$. The latter is clearly well-defined for the values $t(p)-\min \left(\ell_{*}^{-}(p, t), d^{-}(p, t)\right)<t \leq t(p)$.

We now record three statements consistent with the assumptions of Main Theorem I and conclusions of Theorem 2.14.

A1. There exists a constant $c_{\mathcal{N}}=c_{\mathcal{N}}(p) \geq \min \left(\ell_{*}^{-}(p, t), d^{-}(p, t)\right)$ such that $\mathcal{N}^{-}(p)$ has no conjugate terminal points in the time slab $\left[t(p)-c_{\mathcal{N}}, t(p)\right]$.

A2. The metric $\sigma_{t}$ remains close to the metric $\sigma_{0}$, i.e. given any vector $X$ in $T S_{t}$ we have

$$
\left|\sigma_{t}(X, X)-\sigma_{0}(X, X)\right|<\epsilon \sigma_{t}(X, X)
$$

uniformly for all $t(p)-c_{\mathcal{N}} \leq t \leq t(p)$. 
A3. There exists a neighborhood $\mathcal{O}=I_{p} \times U_{p}$ of $p$ and a system of coordinates $x^{\alpha}$ with $x^{0}=t$, the time function introduced above, relative to which the metric $\mathbf{g}$ is close to the Minkowski metric $\mathbf{m}(p)=-n(p)^{2} d t^{2}+\delta_{i j} d x^{i} d x^{j}$,

$$
\left|\mathbf{g}_{\alpha \beta}-\mathbf{m}_{\alpha \beta}(p)\right|<\epsilon
$$

uniformly at all points in $\mathcal{O}$. The set $U_{p}$ contains the cylinder $B_{r_{0}}(p) \times I_{p}$ with the geodesic ball $B_{r_{0}}(p)$ and

$$
\sup _{t \in I_{p}} \pm(t-t(p)) \geq \min \left(\frac{1}{3} r_{0}, d^{ \pm}(t, p)\right) .
$$

In particular, $B_{t, 2(t(p)-t)} \subset \mathcal{O}$ for any $t \in\left[t(p)-\min \left(\frac{1}{3} r_{0}, d^{-}(t, p)\right), t(p)\right]$, where $B_{t, a}$ denotes the Euclidean ball of radius $a$ centered around the point on $\Sigma_{t}$ with the same coordinates $x=\left(x^{1}, x^{2}, x^{3}\right)$ as the point $p$.

Since the constant $\delta_{*}$ is allowed to depend on $r_{0}$ we may assume that $r_{0}>$ $3 \delta_{*}$. As a consequence, given our assumption that $\ell_{*}^{-}(p, t) \leq \min \left(\delta_{*}, d^{-}(p, t)\right)$, we see that the time interval $\left[t(p)-\min \left(\frac{1}{3} r_{0}, d^{-}(t, p)\right), t(p)\right]$ contains the interval $\left[t(p)-\ell_{*}^{-}(p, t), t(p)\right]$,

A4. The space-time region $\mathcal{M}_{I}=\bigcup_{t \in \mathbf{I}} \Sigma_{t}$ contains a future, compact set $\mathcal{D}^{+} \subset$ $\mathcal{M}_{I}$ such that there exists a positive constant $\delta_{0}$ with the property that, for any point $q$ in the complement of $\mathcal{D}^{+}$, we have $\ell_{*}^{-}(q, t)>\min \left(\delta_{0}, d^{-}(p, t)\right)$.

Remark 1. As a first consequence of $\mathbf{A} \mathbf{1}$ we infer that there must exist a largest value of $t \geq t(p)-c_{\mathcal{N}}$ with the property that two distinct null geodesics originating at $p$ intersect at time $t$. Indeed let $t_{0} \geq t(p)-c_{\mathcal{N}}$ be the supremum of such values of $t$ and let $\left(q_{k}, \lambda_{k}, \gamma_{k}\right)$ be a sequence of points $q_{k} \in \mathcal{N}^{-}(p)$ and distinct null geodesics $\lambda_{k}, \gamma_{k}$ from $p$ intersecting at $q_{k}$, with increasing $t\left(q_{k}\right) \geq t(p)-c_{\mathcal{N}}$. By compactness we may assume that $q_{k} \rightarrow q \in \Sigma_{t}, t\left(q_{k}\right) \rightarrow t=t(q)=t_{0}$ and $\lambda_{k} \rightarrow \lambda, \gamma_{k} \rightarrow \gamma$, with both $\lambda, \gamma$ null geodesics passing through $p$ and $q$. We claim that $\gamma \neq \lambda$ and that $q$ is a cut locus terminal point of $\mathcal{N}^{-}(p)$. Indeed if $\gamma \equiv \lambda$, then for a sequence of positive constants $0<\epsilon_{k} \rightarrow 0$ we could find an increasing sequence of indices $k$ such that for null geodesics $\gamma_{k}, \lambda_{k}$ we have that

$$
\mathbf{g}\left(\dot{\gamma}_{k}(0), \dot{\lambda}_{k}(0)\right)=\epsilon_{0}, \quad \gamma_{k}\left(t\left(q_{k}\right)\right)=\lambda_{k}\left(t\left(q_{k}\right)\right), \quad t\left(q_{k}\right) \geq t(p)-c_{\mathcal{N}}
$$

This leads to a contradiction, as by assumption the exponential map $\mathcal{G}^{-}(t, \cdot)$ is a local diffeomorphism for all $t \geq t(p)-c_{\mathcal{N}}$.

Remark 2. As a consequence of $\mathbf{A} \mathbf{2}$ we infer that, for all $t \geq t-c_{\mathcal{N}}$, the distances on $S_{t}$ corresponding to the metrics $\sigma_{t}$ and $\sigma_{0}$ are comparable,

$$
(1-\epsilon) d_{0}\left(q_{1}, q_{2}\right) \leq d_{\sigma}\left(q_{1}, q_{2}\right) \leq(1+\epsilon) d_{0}\left(q_{1}, q_{2}\right), \quad \forall q_{1}, q_{2} \in S_{t},
$$

or, equivalently,

$$
(1+\epsilon)^{-1} d_{\sigma}\left(q_{1}, q_{2}\right) \leq d_{0}\left(q_{1}, q_{2}\right) \leq(1-\epsilon)^{-1} d_{\sigma}\left(q_{1}, q_{2}\right), \quad \forall q_{1}, q_{2} \in S_{t} .
$$

Remark 3. Similarly, as a consequence of A3 the distances on $\Sigma_{t} \cap \mathcal{O}$ corresponding to the induced metric $g$ and the euclidean metric $e$ are also comparable,

$$
(1-\epsilon) d_{e}\left(q_{1}, q_{2}\right) \leq d_{g}\left(q_{1}, q_{2}\right) \leq(1+\epsilon) d_{e}\left(q_{1}, q_{2}\right), \quad \forall q_{1}, q_{2} \in \Sigma_{t} \cap \mathcal{O}, t \in I_{p} .
$$

Observe also that, since $\sigma$ is the metric induced by $g$ on $S_{t}$,

$$
d_{g}\left(q_{1}, q_{2}\right) \leq d_{\sigma}\left(q_{1}, q_{2}\right), \quad \forall q_{1}, q_{2} \in S_{t}, \quad t(p)-\ell_{*}(p)<t \leq t(p) .
$$


Remark 4. In what follows we will assume, without loss of generality, that $t(p)=0$ and that the $x=\left(x^{1}, x^{2}, x^{3}\right)$ coordinates of $p$ are $x=0$. Without loss of generality we may also assume that $n(p)=1$. Indeed if $n(p) \neq 0$ we can rescale the time variable $t=\tau / n(p)$ such that relative to the new time we have $\mathbf{g}=-\frac{n^{2}}{n(p)^{2}} d \tau^{2}+$ $g_{i j} d x^{i} d x^{j}$. Once we find a convenient value for $\delta_{*}^{\prime}$ such that no distinct past null geodesics from $p$ intersect for values of $|\tau| \leq \delta_{*}^{\prime}$, we find the desired $\delta_{*}=\delta_{*}^{\prime} \cdot n(p) \geq$ $\delta_{*}^{\prime} N_{0}^{-1}$.

According to Remark 1 we can find a largest value of time $t_{*}=t(p)-\ell_{*}^{-}(p, t)<$ $t(p)$ where two distinct null geodesics from $p$ intersect, say at point $q$ with $t(q)=t_{*}$. Our next result will imply that at $q$ the angle between projections 13 of $\dot{\gamma}_{1}\left(t_{*}\right)$ and $\dot{\gamma}_{2}\left(t_{*}\right)$ onto $T_{q} \Sigma_{t_{*}}$ is precisely $\pi$.

Lemma 3.1. Let $t_{*}=t_{*}(p)<0$ be the larges 14 value of $t$ such that that there exist two distinct past directed null geodesics $\gamma_{1}, \gamma_{2}$ from $p$ intersecting at $q$ with $t(q)=t_{*}$. Assume also that the exponential map $\mathcal{G}^{-}=(t, \omega) \rightarrow \gamma_{\omega}(t)$ is a global diffeomorphism from $\left(t_{*}(p), t(p)\right] \times \mathbb{S}^{2}$ to its image on $\mathcal{N}^{-}(p)$ and a local diffeomorphism 15 on $\left[t_{*}(p), t(p)\right] \times \mathbb{S}^{2}$. Then, at $q$, the projections of $\dot{\gamma}_{1}\left(t_{*}\right)$ and $\dot{\gamma}_{2}\left(t_{*}\right)$ onto $T_{q} \Sigma_{t_{*}}$ belong to the same line and point in opposite directions.

Remark 5. A similar statement also holds for future directed null geodesics.

Proof. The distinct null geodesics $\gamma_{1}, \gamma_{2}$ can be identified with the null geodesics $\gamma_{\omega_{1}}, \gamma_{\omega_{2}}$ with $\omega_{1} \neq \omega_{2} \in \mathbb{S}^{2}$, two distinct directions in the tangent space $T_{p} \mathcal{M}$.

By assumptions $\gamma_{\omega_{1}}\left(t_{*}\right)=\gamma_{\omega_{2}}\left(t_{*}\right)=q$ and there exist disjoint neighborhoods $\mathcal{V}_{1}$ of $\left(t_{*}, \omega_{1}\right)$ and $\mathcal{V}_{2}$ of $\left(t_{*}, \omega_{2}\right)$ in $\mathbb{R}_{-} \times \mathbb{S}^{2}$ such that the restrictions of $\mathcal{G}$ to $\mathcal{V}_{1}$, $\mathcal{V}_{2}$ are both diffeomorphisms. We can choose both neighborhoods to be of the form $\mathcal{V}_{i}=\left(t_{*}-\epsilon, t_{*}\right] \times W_{i}$ with $\omega_{i} \in W_{i}$ for $i=1,2$. Let $\mathcal{G}_{t}(\omega)=\mathcal{G}(t, \omega)$ and define $S_{t, i}=\mathcal{G}_{t}\left(W_{i}\right), i=1,2$. They are both pieces of embedded 2-surfaces in $\Sigma_{t}$, $t \in\left(t_{*}-\epsilon_{0}, t_{*}\right]$, for some $\epsilon_{0}>0$, and, as the exponential map $\mathcal{G}(t, \cdot)$ is assumed to be a global diffeomorphism for any $t>t_{*}$, they are disjoint for all $t>t_{*}$.

For $t=t_{*}$ the surfaces $S_{t_{*}, i}$ intersect at the point $q$. We claim that the tangent spaces of $T_{q}\left(S_{t_{*}, 1}\right)$ and $T_{q}\left(S_{t_{*}, 2}\right)$ must coincide in $T_{q}\left(\Sigma_{*}\right)$. Otherwise, since $T_{q}\left(S_{t_{*}, 1}\right)$ and $T_{q}\left(S_{t_{*}, 2}\right)$ are two-dimensional hyperplanes in a three-dimensional space $T_{q} \Sigma_{t_{*}}$, they intersect transversally, and by an implicit function we conclude that the surfaces $S_{t_{*}, i}$ also intersect transversally at $q$. The latter is impossible, as $S_{t, 1}, S_{t, 2}$ are continuous families of 2-surfaces disjoint for all $t>t_{*}$.

The following lemma is a consequence of $\mathbf{A 3}$ and the normalization made in Remark 4. Recall that $\mathcal{I}^{-}(p)$ denotes the causal past of point $p$, i.e. it consists of all points which can be reached by continuous, past time-like curves from $p$, and $\mathcal{N}^{-}(p)$ is the boundary of $\mathcal{I}^{-}(p)$.

Lemma 3.2. Let $t \in\left[-\min \left(\frac{1}{3} r_{0}, d^{-}(t, p)\right), 0\right]$ and and let $p_{t}$ be the point on $\Sigma_{t}$ which has the same coordinates $x=\left(x^{1}, x^{2}, x^{3}\right)=0$ as $p$. Let $B_{t, r}=B\left(p_{t}, r\right) \subset \Sigma_{t}$ be the euclidean ball centered at $p_{t}$ of radius $r$ and $B_{r}^{c}$ its complement in $\Sigma_{t}$. Then,

$$
B_{t,(1-3 \epsilon)|t|} \subset \mathcal{I}^{-}(p) \cap \Sigma_{t}, \quad B_{t,(1+3 \epsilon)|t|}^{c} \cap\left(\mathcal{I}^{-}(p) \cup \mathcal{N}^{-}(p)\right)=\emptyset .
$$

\footnotetext{
${ }^{13}$ Defined relative to the decomposition $X=X^{0} \mathbf{T}+\underline{X}$, where $\underline{X} \in T_{q} \Sigma_{t_{*}}$.

${ }^{14}$ We assume that such a point exists.

${ }^{15}$ Local with respect to angles on $\mathbb{S}^{2}$.
} 
Proof. According to Remark $4 p$ has coordinates $t=0, x=0$ and $n(p)=1$. Hence, according to (21), $|n-1|<\epsilon$ and $\left|g_{i j}-\delta_{i j}\right|<\epsilon$. The point $p_{t}$ has coordinates $(t, 0), t>-r_{0} / 3$. Let $q \in B_{t,(1-2 \epsilon)|t|}$ of coordinate $(t, y)$ and $\ell(\tau)=\left(\tau, y \frac{\tau}{t}\right)$ be the straight segment connecting $p$ with $q$. Thus, in view of (21), for sufficiently small $\epsilon$,

$$
\begin{aligned}
\mathbf{g}(\dot{\ell}(\tau), \dot{\ell}(\tau)) & =\mathbf{m}(\dot{\ell}(\tau), \dot{\ell}(\tau))+(\mathbf{g}-\mathbf{m})(\dot{\ell}(\tau), \dot{\ell}(\tau)) \\
& \leq\left(-1+\frac{|y|^{2}}{t^{2}}\right)+\epsilon\left(1+\frac{|y|^{2}}{t^{2}}\right)=-1+\epsilon+(1+\epsilon) \frac{|y|^{2}}{t^{2}} \\
& <-1+\epsilon+(1+\epsilon)(1-2 \epsilon)^{2}=-2 \epsilon+O(\epsilon)^{2}<0 .
\end{aligned}
$$

Thus $q$ can be reached by a time-like curve from $p$; therefore $q \in \mathcal{J}^{-}(p)$.

On the other hand, if $\ell(\tau)=(\tau, x(\tau))$ is an arbitrary causal curve from $p$, then

$$
\begin{aligned}
0 \geq \mathbf{g}(\dot{\ell}(\tau), \dot{\ell}(\tau)) & =\mathbf{m}(\dot{\ell}(\tau), \dot{\ell}(\tau))+(\mathbf{g}-\mathbf{m})(\dot{\ell}(\tau), \dot{\ell}(\tau)) \\
& \geq\left(-1+|\dot{x}|^{2}\right)-\epsilon\left(1+|\dot{x}|^{2}\right)=-(1+\epsilon)+(1-\epsilon)|\dot{x}|^{2} .
\end{aligned}
$$

Therefore $|\dot{x}| \leq\left(\frac{1+\epsilon}{1-\epsilon}\right)^{1 / 2}<1+2 \epsilon+O\left(\epsilon^{2}\right)$ and thus, for sufficiently small $\epsilon>0$, $|x(\tau)| \leq(1+3 \epsilon) \tau$. Consequently points $q$ in the complement of the ball $B_{t,(1+3 \epsilon)|t|}$ cannot be reached from $p$ by a causal curve.

Corollary 3.3. Let $t \in\left[-\min \left(\frac{1}{3} r_{0}, d^{-}(t, p)\right), 0\right]$. Any continuous curve $x(\tau) \subset \Sigma_{t}$ between two points $q_{1} \in B_{t,(1-3 \epsilon)|t|}$ and $q_{2} \in B_{t, r(1+3 \epsilon)|t|}^{c}$ has to intersect $\mathcal{N}^{-}(p) \cap \Sigma_{t}$.

Proof. This is an immediate consequence of Lemma 3.2 and the fact that both $\mathcal{I}^{-}(p)$ and $\left(\mathcal{I}^{-}(p) \cup \mathcal{N}^{-}(p)\right)^{c}$ are connected open, disjoint, sets.

Remark 6. Observe that the argument used in the proof of Lemma 3.2 also shows the inclusion

$$
\mathcal{N}^{-}(p) \cap \Sigma_{t} \subset B_{t,(1+3 \epsilon)|t|} \cap B_{t,(1-3 \epsilon)|t|}^{c}, \quad \forall t:-\min \left(\frac{1}{3} r_{0}, d^{-}(t, p)\right) \leq t \leq 0 .
$$

We are now ready prove the following.

Proposition 3.4. Assume A1, A2, and $\mathbf{A 3}$ are satisfied and

$$
\ell_{*}^{-}(p, t) \leq \min \left(\delta_{*}, d^{-}(p, t)\right)
$$

with $r_{0}>3 \delta_{*}$. Then, no two null geodesics originating at $p$ and also opposit $\mathbb{1 6}^{16}$ at $p$ can intersect in the slab $\left[t(p)-\ell_{*}(p, t), t(p)\right)$.

Remark. Modulo the assumption that the intersecting geodesics have to be opposite at $p$, Proposition 3.4 gives the desired contradiction and implies Main Theorem I. Indeed, Remark 1 implies that if $\ell_{*}^{-}(p, t) \leq \min \left(\delta_{*}, d^{-}(p, t)\right)$, then there exist two distinct null geodesics from $p$ necessarily intersecting at time $t(p)-\ell_{*}^{-}(p, t)$, which contradict the above proposition. The extra assumption that the geodesics are opposite at $p$ will be settled below by showing the existence of a point $p \in \mathcal{M}_{I}$ with the property that there exist two null geodesics from $p$ intersecting precisely at time $t(p)-\ell_{*}^{-}(p, t)$, which are also opposite at $p$.

\footnotetext{
${ }^{16}$ I.e. two null geodesics $\gamma_{1}, \gamma_{2}$ with the property that $\gamma_{1}(0)=\gamma_{2}(0)=p$ and the projections of the tangent vectors $\dot{\gamma}_{1}(0), \dot{\gamma}_{2}(0)$ to $T_{p} \Sigma_{t(p)}$ belong to the same line and point in opposite directions.
} 
Proof. Once again we set $t(p)=x(p)=0$ and $n(p)=1$. We now argue by contradiction. Assume that there exist two opposite null geodesics $\gamma_{1} \neq \gamma_{2}$ from $p$ such that $\gamma_{1}\left(t_{*}, \omega_{1}\right)=\gamma_{2}\left(t_{*}, \omega_{2}\right)$, where $t_{*}$ is the first time of intersection of all such geodesics, with $t_{*} \geq-\ell_{*}(p, t)$. We choose the time $t_{0}>t_{*}$ such that the distance $d_{g}\left(\gamma_{1}\left(t_{0}, \omega_{1}\right), \gamma_{2}\left(t_{0}, \omega_{2}\right)\right)<\left|t_{0}\right| \epsilon / 2$. Let $q_{1}=\gamma_{1}\left(t_{0}, \omega_{1}\right)$ and $q_{2}=\gamma_{2}\left(t_{0}, \omega_{2}\right)$. Note that by our assumptions the exponential map $\mathcal{G}^{-}(t, \cdot)$ is a global diffeomorphism for all $0<t \leq t_{0}$. Our assumption also implies that $\omega_{1}$ and $\omega_{2}$ represent antipodal points on $\mathbb{S}^{2}$.

We consider the set

$$
\Omega=\left\{\omega \in \mathbb{S}^{2}: d_{\mathbb{S}^{2}}\left(\omega, \omega_{2}\right)<\frac{\pi}{4}\right\}=\left\{\omega \in \mathbb{S}^{2}: d_{\mathbb{S}^{2}}\left(\omega, \omega_{1}\right) \geq \frac{3 \pi}{4}\right\} .
$$

Then, in view of (22) and (25), the set $\tilde{\Omega}_{t_{0}}=\mathcal{G}^{-}\left(t_{0}, \Omega\right)$ has the property that 17

$$
d_{g}\left(q_{2}, q\right) \leq d_{\sigma}\left(q_{2}, q\right)<(1+\epsilon) d_{0}\left(q_{2}, q\right)=(1+\epsilon)\left|t_{0}\right| \frac{\pi}{4}, \quad \forall q \in \tilde{\Omega}_{t_{0}} .
$$

Thus, in view of $d_{g}\left(q_{1}, q_{2}\right)<t_{0} \epsilon / 2$ and the triangle inequality,

$$
d_{g}\left(q_{1}, q\right) \leq(1+\epsilon)\left|t_{0}\right| \frac{\pi}{4}+\frac{\epsilon}{2} t_{0} \leq \frac{\pi}{4}\left|t_{0}\right|(1+O(\epsilon)), \quad \forall q \in \tilde{\Omega}_{t_{0}} .
$$

Thus, taking into account (24),

$$
d_{e}\left(q_{1}, q\right) \leq \frac{1}{1-\epsilon} d_{g}\left(q_{1}, q\right) \leq \frac{\pi}{4}\left|t_{0}\right|(1+O(\epsilon)), \quad \forall q \in \tilde{\Omega}_{t_{0}} .
$$

On the other hand, from (22), since any point in the complement of $\tilde{\Omega}_{t_{0}}$ in $\mathcal{N}^{-}(p) \cap$ $\Sigma_{t_{0}}$ lies in the image by $\mathcal{G}^{-}$of the complement of $\Omega \subset \mathbb{S}^{2}$,

$$
d_{\sigma}\left(q_{1}, q\right) \leq(1+\epsilon) d_{0}\left(q_{1}, q\right) \leq \frac{3 \pi}{4}(1+\epsilon)\left|t_{0}\right|, \quad \forall q \in\left(\mathcal{N}^{-}(p) \cap \Sigma_{t_{0}}\right) \backslash \tilde{\Omega} .
$$

Observe that, since $q_{1} \in \mathcal{N}^{-}(p) \cap \Sigma_{t_{0}}$ and using (27), we have $q_{1} \in B_{t_{0},(1+3 \epsilon)\left|t_{0}\right|} \cap$ $B_{t_{0},(1-3 \epsilon)\left|t_{0}\right|}^{c}$. Thus if $\left(t_{0}, y\right)$ are the coordinates of $q_{1}$ we must have

$$
1-3 \epsilon \leq \frac{|y|}{\left|t_{0}\right|} \leq 1+3 \epsilon
$$

Thus, for sufficiently small $\epsilon>0$, the point $\left(t_{0},-\left(1-7 \epsilon\left|t_{0}\right|\right) y\right) \in B_{t_{0},(1-3 \epsilon)\left|t_{0}\right|}$ while $\left(t_{0},-\left(1+7 \epsilon\left|t_{0}\right|\right) y\right) \in B_{t_{0},(1+3 \epsilon)\left|t_{0}\right|}^{c}$. Let $y(\tau)=-\left(14 \epsilon \tau+\left(1-7 \epsilon\left|t_{0}\right|\right)\right) \cdot y$, with $\tau \in[0,1]$ and $I$ the segment $I(\tau)=\left(t_{0}, y(\tau)\right)$. Observe that all points of $I$ are within euclidean distance $O\left(\epsilon\left|t_{0}\right|\right)$ from the point $q^{o p p}=\left(t_{0},-y\right)$. Clearly, the extremities of $I$ verify $I(0) \in B_{t_{0},(1-3 \epsilon)\left|t_{0}\right|}$ and $I(1) \in B_{t_{0},(1+3 \epsilon)\left|t_{0}\right|}^{c}$. To reach a contradiction with Corollary 3.3 we will show that $I$ does not intersect $\mathcal{N}^{-}(p) \cap \Sigma_{t_{0}}$.

We first show that $I \cap \tilde{\Omega}_{t_{0}}=\emptyset$. Indeed, if $q \in \tilde{\Omega}_{t_{0}}$, we have, from (29),

$$
d_{e}\left(q_{1}, q\right) \leq \frac{\pi}{4}\left|t_{0}\right|(1+O(\epsilon))<\left|t_{0}\right|,
$$

while, if $q \in I$,

$$
d_{e}\left(q_{1}, q\right) \geq|y|\left(2-7 \epsilon\left|t_{0}\right|\right) \geq\left|t_{0}\right|(1-3 \epsilon)\left(2-7 \epsilon\left|t_{0}\right|\right)>\left|t_{0}\right| .
$$

\footnotetext{
${ }^{17}$ Recall that $d_{0}$ denotes the distance function on $S_{t}$ defined with respect to the metric $\sigma_{0}$ obtained by pushing forward the standard metric on $\mathbb{S}^{2}$ by the exponential map $\mathcal{G}^{-}(t, \cdot)$.
} 
Now, assume by contradiction, the existence of $q \in\left(\left(\mathcal{N}^{-}(p) \cap \Sigma_{t_{0}}\right) \backslash \tilde{\Omega}_{t_{0}}\right) \cap I$. From (30) we must infer that

$$
d_{\sigma}\left(q_{1}, q\right) \leq \frac{3 \pi}{4}(1+\epsilon)\left|t_{0}\right|
$$

On the other hand let $x(\tau), \tau \in[0,1]$, be the $\sigma$-geodesic connecting $q_{1}$ and $q$ in $S_{t_{0}}$. Since $S_{t_{0}}=\mathcal{N}^{-}(p) \cap \Sigma_{t_{0}}$ is contained in the set $B_{t_{0},(1+3 \epsilon)\left|t_{0}\right|} \cap B_{t_{0},(1-3 \epsilon)\left|t_{0}\right|}^{c}$, so is the entire curve $x(\tau)$ for all $0 \leq \tau \leq 1$. Now observe that the euclidean distance of any curve which connects $q$ and $q^{o p p}$ while staying outside $B_{t_{0},(1-3 \epsilon)\left|t_{0}\right|}$ must be greater than $\pi(1-3 \epsilon)\left|t_{0}\right|$. Since all points in $I$ are within euclidean distance $O\left(\epsilon\left|t_{0}\right|\right)$ from $q^{o p p}$ we infer that

$$
\int_{0}^{1}|\dot{x}(\tau)|_{e} d \tau \geq \pi\left|t_{0}\right|(1-O(\epsilon))
$$

This implies that

$$
d_{\sigma}\left(q_{1}, q\right)=\int_{0}^{1}|\dot{x}(\tau)|_{g} d \tau \geq(1-\epsilon) \int_{0}^{1}|\dot{x}(\tau)|_{e} d \tau \geq \pi\left|t_{0}\right|(1-O(\epsilon)),
$$

which is a contradiction. Thus $I$ does not intersect $\mathcal{N}^{-}(p) \cap \Sigma_{t_{0}}$.

The proof of Proposition 3.4 depends on the fact that the intersecting null geodesics from $p$ are opposite to each other in the tangent space $T_{p}\left(\Sigma_{t(p)}\right)$. According to Lemma 3.1 we know that, at the first time $t$ when two past directed null geodesics $\gamma, \lambda$ from $p$ intersect at a point $q$, they must intersect opposite to each other. However the situation is not entirely symmetric, as there may exist another pair of future directed null geodesics $\gamma^{\prime}, \lambda^{\prime}$ from $q$ which intersect at a point $p_{1}$ with $t\left(p_{1}\right)$ strictly smaller than $t(p)$. We can then repeat the procedure with $p$ replaced by $p_{1}$ and with a new pair of null geodesics $\gamma_{1}, \lambda_{2}$ from $p_{1}$ intersecting at $q_{1}$ with $t\left(q_{1}\right)$ the smallest value of $t$ such that any two null geodesics from $p_{1}$ intersect on $\Sigma_{t}$. Proceeding by induction we can construct a sequence of points $p_{k}$, $q_{k}$ with $t\left(p_{k}\right)$ monotonically decreasing and $t\left(q_{k}\right)$ monotonically increasing, and a sequence of pairs of distinct null geodesics $\gamma_{k}, \lambda_{k}$ passing through both $p_{k}$ and $q_{k}$. Our construction also insures that at $q_{k}$ the geodesics $\gamma_{k}, \lambda_{k}$ are opposite to each other. We would like to pass to limit and thus obtain two null geodesics which intersect each other at two distinct points. This procedure is behind the proof of the following

Proposition 3.5. Assume that the region $\mathcal{M}_{I}$ verifies $\mathbf{A 4}$. Then, if there exist two distinct null geodesics $\lambda_{0}, \gamma_{0}$, in $\mathcal{M}_{I}$, intersecting at two points $p_{0}, q_{0}$ such that $0<t\left(p_{0}\right)-t\left(q_{0}\right) \leq \min \left(\delta_{*}, d^{-}\left(p_{0}, t\left(p_{0}\right)\right)\right)$, then there must exist a pair of null geodesics $\lambda, \gamma$ intersecting at points $p, q$ with $t\left(q_{0}\right) \leq t(q)<t(p) \leq t\left(p_{0}\right)$ which are opposite at both $p$ and $q$.

Proof. Let

$$
\Delta t:=\min _{p, q \in \mathcal{M}_{I}} t(p)-t(q)
$$

such that there exists a pair of distinct past directed null geodesics originating at $p$ and intersecting at $q$, where $t(p) \leq t\left(p_{0}\right)$ and $t(q) \geq t\left(q_{0}\right)$. By the assumption of the proposition $\Delta t \leq \min \left(\delta_{*}, d^{-}\left(p_{0}, t\left(p_{0}\right)\right)\right)$. On the other hand, for all points $p \in \mathcal{M}_{I} \backslash \mathcal{D}^{+}$, where the set $\mathcal{D}^{+}$is that of condition $\mathbf{A 4}$, we have that $\ell_{*}^{-}(p, t)>$ $\min \left(\delta_{0}, d^{-}(p, t)\right)$. Assuming, without loss of generality, that $\delta_{*}<\delta_{0}$, we see that it 
suffices to impose the restriction $p \in \mathcal{D}^{+}$in (31). Since $\mathcal{D}^{+} \cap\left[t\left(q_{0}\right), t\left(p_{0}\right)\right]$ is compact and the manifold $\mathcal{M}_{I}$ is smooth, we can conclude that $\Delta t>0$.

Let $p_{n} \in \mathcal{D}^{+} \cap\left[t\left(q_{0}\right), t\left(p_{0}\right)\right]$ be a sequence of points such that $\ell_{*}\left(p_{n}, t\right) \rightarrow \Delta t$. For each $p_{n}$ we may assume, with the help of Theorem 2.14, that A1-A4 are satisfied for $p_{n}$.

Choosing a subsequence, if necessary, we can assume that $p_{n} \rightarrow p$. We claim that $\ell_{*}(p, t)=\Delta t$, i.e. there exists a pair of distinct past null geodesics from $p$ intersecting at time $t(p)-\Delta t$, and that these geodesics are opposite to each other at $p$. First, to show the existence of such geodesics we assume, by contradiction, that no two distinct geodesics from $p$ intersect at $t \geq t(p)-\Delta t$. Since by assumption $\Delta t<$ $\delta_{*}$ and, clearly $\Delta t \leq d^{-}(p, t)$, we may assume that $\mathcal{N}^{-}(p)$ does not contain points conjugate to $p$ in the slab $[t(p)-\Delta t, t(p)]$. This implies that the exponential map $\mathcal{G}_{p}^{-}(t, \cdot)$ is a global diffeomorphism for all $t \in[t(p)-\Delta t, t(p))$. Smooth dependence of the exponential map $\mathcal{G}_{q}$ on the base point $q$ implies that there exists a small neighborhood $\mathcal{U}$ of $p$ such that for any $q \in \mathcal{U}$ the exponential map $\mathcal{G}_{q}^{-}(t, \cdot)$ is a global diffeomorphism for any $t \in\left[t(q)-\min \left(\Delta t+\epsilon_{0}, d^{-}(q, t)\right), t(q)\right)$ for some $\epsilon_{0}>0$. This however contradicts the existence of our sequence $p_{n} \rightarrow p$ since by construction $\ell_{*}\left(p_{n}, t\right) \rightarrow \Delta t$.

Therefore we may assume that there exists a pair of null geodesics $\gamma_{1}, \gamma_{2}$, originating at $p$ and intersecting at a point $q$ with $t(q)=t(p)-\Delta t=t(p)-\ell_{*}(p, t)$. By Lemma 3.1 the geodesics $\gamma_{1}$ and $\gamma_{2}$ are opposite at $q$. We need to show that they are also opposite at $p$. Consider the boundary $\mathcal{N}^{+}(q)$ of the causal future of $q$. It contains a pair of null geodesics, the same $\gamma_{1}$ and $\gamma_{2}$, intersecting at $p$. Thus, either $t(p)$ is the first time of intersection among all distinct future directed null geodesics from $q$, in which case Lemma 3.1 implies that $\gamma_{1}$ and $\gamma_{2}$ are opposite at $p$, or there exists a pair of null geodesics from $q$ intersecting at a point $p^{\prime}$ such that $t\left(p^{\prime}\right)<t(p)$. But then $t\left(p^{\prime}\right)-t(q)<\Delta t$, contradicting the definition of $\Delta t$.

\section{Proof of Main Theorem II}

We start with the following proposition.

Proposition 4.1. Assume (5), (6) are verified. Then, if the initial metric $g$ on $\Sigma_{0}$ verifies (7), there exists a large constant $C=C\left(N_{0}, \mathcal{K}_{0}, I_{0}\right)$ such that, relative to the induced transported coordinates in $I \times U$ we have,

$$
C^{-1}|\xi|^{2} \leq g_{i j}(t, x) \xi^{i} \xi^{j} \leq C|\xi|^{2}, \quad \forall x \in U .
$$

Proof. We fix a coordinate chart $U$ and consider the transported coordinates $t, x^{1}$, $x^{2}, x^{3}$ on $I \times U$. Thus $\partial_{t} g_{i j}=-\frac{1}{2} n k_{i j}$. Let $X$ be a time-independent vector on $\mathbf{M}$ tangent to $\Sigma_{t}$. Then,

$$
\partial_{t} g(X, X)=-\frac{1}{2} n k(X, X)
$$

Clearly,

$$
|n k(X, X)| \leq|n k|_{g}|X|_{g}^{2} \leq\|n k(t)\|_{L^{\infty}}|X|_{g}^{2}
$$

with $|k|_{g}^{2}=g^{a c} g^{b d} k_{a b} k_{c d}$ and $|X|_{g}^{2}=X^{i} X^{j} g_{i j}=g(X, X)$. Therefore, since $\partial_{t}|X|_{g}^{2}=$ $\partial_{t} g(X, X)$,

$$
-\frac{1}{2}\|n k(t)\|_{L^{\infty}}|X|_{g}^{2} \leq \partial_{t}|X|_{g}^{2} \leq \frac{1}{2}\|n k(t)\|_{L^{\infty}}|X|_{g}^{2}
$$


Thus,

$$
|X|_{g_{0}} e^{-\int_{t_{0}}^{t}\|n k(\tau)\| d \tau} \leq|X|_{g_{t}}^{2} \leq|X|_{g_{0}} e^{\int_{t_{0}}^{t}\|n k(\tau)\| d \tau}
$$

from which (32) immediately follows.

Corollary 4.2. Let $p \in \Sigma_{t}$ in a coordinate chart $U_{t}=\Sigma_{t} \cap(I \times U)$ with transported coordinates $\left(t, x^{1}, x^{2}, x^{3}\right)$. Denote by $e$ the euclidean metric on $U_{t}$ relative to the coordinates $x=\left(x^{1}, x^{2}, x^{3}\right)$. Let $B_{r}^{(e)}(p) \subset U_{t}$ be a euclidean ball of radius $r$ centered at $p$. Then, for all $\rho \geq C r$, with $C=C\left(N_{0}, \mathcal{K}_{0}, I_{0}\right)$ the constant of Proposition 4.1, the euclidean ball $B_{r}^{(e)}(p)$ is included in the geodesic balls $B_{\rho}(p)$, relative to the metric $g_{t}$,

$$
B_{r}^{(e)}(p) \subset B_{\rho}(p), \quad \rho \geq C r .
$$

Proof. Let $q \in B_{r}^{(e)}(p)$ and $\gamma:[0,1] \rightarrow B_{r}^{(e)}(p)$ be the line segment between $p$ and q. Clearly, in view of (32),

$$
\operatorname{dist}_{e}(p, q)=\int_{0}^{1}(e(\dot{\gamma}, \dot{\gamma}))^{\frac{1}{2}} d \tau \geq C^{-1} \int_{0}^{1}\left(g_{t}(\gamma(\tau))(\dot{\gamma}, \dot{\gamma})\right)^{\frac{1}{2}} d \tau \geq C^{-1} \operatorname{dist}_{g_{t}}(p, q) .
$$

Thus for any $q \in B_{r}^{(e)}(p)$ we have $\operatorname{dist}_{g_{t}}(p, q) \leq C \operatorname{dist}_{e}(p, q) \leq C r$. Therefore $q$ belongs to the geodesic ball $B_{\rho}(p)$ for any $\rho \geq C r$, as desired.

The corollary allows us to get a lower bound for the volume radius. We recall below the definition of volume radius on a general Riemannian manifold $M$. The Corollary allows us to get a lower bound for the volume radius. We recall below the definition of volume radius on a general Riemannian manifold $M$.

Definition 4.3. The volume radius $r_{v}(p, \rho)$ at point $p \in M$ and scales $\leq \rho$ is defined by

$$
r_{v o l}(p, \rho)=\inf _{r \leq \rho} \frac{\left|B_{r}(p)\right|}{r^{3}},
$$

with $\left|B_{r}\right|$ the volume of $B_{r}$ relative to the metric $g$. The volume radius $r_{v o l}(M, \rho)$ of $M$ on scales $\leq \rho$ is the infimum of $r_{v o l}(p, \rho)$ over all points $p \in M$.

Let $\rho_{0}$ be the positive number of the initial assumption I1. Thus every point $p \in \Sigma_{t}$ belongs to a euclidean ball $B_{\rho_{0}}^{(e)}(p)$, relative to local transported coordinates. Let $B_{r}(p)$ be a geodesic ball around $p$. According to Corollary 4.2 for any $a \leq$ $\min \{\rho, r / C\}$ we must have $B_{a}^{(e)}(p) \subset B_{r}(p)$. Therefore, according to Proposition 4.1 .

$$
\left|B_{r}(p)\right|_{g_{t}} \geq\left|B_{a}^{(e)}(p)\right|_{g_{t}}=\int_{B_{a}^{(e)}(p)} \sqrt{\left|g_{t}\right|} d x \geq C^{-3 / 2}\left|B_{a}^{(e)}(p)\right|_{e} \geq C^{-3 / 2} a^{3} .
$$

This means that, for all $r \leq C \rho$,

$$
\left|B_{r}(p)\right| \geq C^{-3 / 2}(r / C)^{3} .
$$

Thus, on scales $\rho^{\prime} \leq C \rho, \rho \leq \rho_{0}$, we must have $r_{v o l}\left(p, \rho^{\prime}\right) \geq C^{-9 / 2}$. Choosing $\rho \leq \rho_{0}$ such that $C \rho=1$, we deduce the following,

Proposition 4.4. Under the assumptions I1 as well as (5), (6), there exists a sufficiently small constant $v=v\left(I_{0}, \rho_{0}, N_{0}, \mathcal{K}_{0}\right)>0$, depending only on $I_{0}, \rho_{0}, N_{0}$, $\mathcal{K}_{0}$, such that the volume radius of each $\Sigma_{t}$, for scales $\leq 1$, is bounded from below:

$$
r_{v o l}\left(\Sigma_{t}, 1\right) \geq v \text {. }
$$


We rely on Proposition 4.4 to prove the existence of good local space-time coordinates on $\mathcal{M}_{I}$. The key to our construction is the following general result, based on Cheeger-Gromov convergence of Riemannian manifolds; see A2 or Theorem 5.4. in $\mathrm{Pe}$.

Theorem 4.5. Given $\Lambda>0, v>0$ and $\epsilon>0$ there exists an $r_{0}>0$ such that any 3-dimensional, complete, Riemannian manifold $(M, g)$ with $\|R\|_{L^{2}} \leq \Lambda$ and volume radius, at scales $\leq 1$ bounded from below by $v$, i.e. $r_{v o l}(M, 1) \geq v$, verifies the following property:

Every geodesic ball $B_{r}(p)$, with $p \in M$ and $r \leq r_{0}$ admits a system of harmonic coordinates $x=\left(x^{1}, x^{2}, x^{3}\right)$ relative to which we have

$$
\begin{aligned}
(1+\epsilon)^{-1} \delta_{i j} \leq g_{i j} & \leq(1+\epsilon) \delta_{i j}, \\
r \int_{B_{r}(p)}\left|\partial^{2} g_{i j}\right|^{2} d v_{g} & \leq \epsilon .
\end{aligned}
$$

We apply this theorem to the family of complete Riemannian manifolds $\left(\Sigma_{t}, g_{t}\right)_{t \in I}$, for $p=2$. According to Proposition 4.4 we have a uniform lower bound for the volume radius $r_{v o l}\left(\Sigma_{t}, 1\right)$. On the other hand we also have a uniform bound on the $L^{2}$ norm of the Ricci curvature tensor 19 Indeed, according to Proposition 5.2 of the next section, there exists a constant $C=C\left(N_{0}, \mathcal{K}_{0}, I_{0}\right)$ such that, for any $t \in I$,

$$
\|\mathbf{R}(t)\|_{L^{2}} \leq C\left(N_{0}, \mathcal{R}_{0}\right)\left\|\mathbf{R}\left(t_{0}\right)\right\|_{L^{2}}=C \mathcal{R}_{0} .
$$

Therefore, for any $\epsilon>0$, there exists $r_{0}$ depending only on $\epsilon, I_{0}, \rho_{0}, N_{0}, \mathcal{K}_{0}, R_{0}$ such that on any geodesic ball, $B_{r} \subset \Sigma_{t}, r \leq r_{0}$, centered at a point $p_{t} \in \Sigma_{t}$, there exist local coordinates relative to which the metric $g_{t}$ verifies conditions (33)-(34). Starting with any such coordinate system $x=\left(x^{1}, x^{2}, x^{3}\right)$ we consider a cylinder $J \times B_{r}$ with $J=(t-\delta, t+\delta) \cap I$ and the associated transported coordinates $(t, x)$ for which (2) holds, i.e.

$$
\mathbf{g}=-n^{2} d t^{2}+g_{i j} d x^{i} d x^{j} .
$$

Integrating equation (3) and using assumptions (5), (6) we derive, for all $t^{\prime} \in J$ and $\delta$ sufficiently small,

$$
\begin{aligned}
\left|g_{i j}\left(t^{\prime}, x\right)-g_{i j}(t, x)\right| & \leq 2 \int_{J}\|n k(s)\|_{L^{\infty}} d s \leq 2 N_{0}|J| \sup _{t \in J}\|k(t)\|_{L^{\infty}} \\
& \leq 2 N_{0} \frac{|J|}{|I|} \mathcal{K}_{0} \leq \epsilon,
\end{aligned}
$$

provided that $4 \delta|I|^{-1} N_{0} \mathcal{K}_{0}<\epsilon$. On the other hand, according to (33) we have for all $x \in B_{r}$,

$$
\left|g_{i j}(t, x)-\delta_{i j}\right| \leq \epsilon .
$$

Therefore, for a sufficiently small interval $J$, whose size $2 \delta$ depends only on $N_{0}, \mathcal{K}_{0}$ and $\epsilon>0$, we have, for all $\left(t^{\prime}, x\right) \in J \times B_{r}$,

$$
\left|g_{i j}\left(t^{\prime}, x\right)-\delta_{i j}\right| \leq 2 \epsilon .
$$

\footnotetext{
${ }^{18} \mathrm{An}$ appropriate version of the theorem holds in every dimension $N$ with an $L^{p}$ bound of the Riemann curvature tensor and $p>N / 2$.

${ }^{19}$ Which coincides with the full Riemann curvature tensor in three dimensions.
} 
On the other hand assumption (6) also provides us with a bound for $\partial_{t} \log n$, i.e. $|I| \cdot \sup _{t \in I}\left\|\partial_{t} \log n(t)\right\|_{L^{\infty}} \leq \mathcal{K}_{0}$. Hence also,

$$
|J| \sup _{t \in J}\left\|\partial_{t} n(t)\right\|_{L^{\infty}} \leq N_{0}^{-1} \frac{|I|}{|J|} \mathcal{K}_{0} .
$$

Therefore, with a similar choice of $|J|=2 \delta$ we have

$$
\left|n\left(t^{\prime}, x\right)-n(t, x)\right| \leq 2 \delta N_{0}^{-1} \frac{|I|}{|J|} \mathcal{K}_{0}<\epsilon .
$$

Now, let $n(p)$ be the value of the lapse $n$ at the center $p$ of $B_{r} \subset \Sigma_{t}$. Clearly, for all $x \in B_{r}$,

$$
|n(t, x)-n(p)| \leq r\|\nabla n\|_{L^{\infty}\left(B_{r}\right)} \leq r N_{0}^{-1}\|\nabla \log n\|_{L^{\infty}\left(B_{r}\right)} \leq r N_{0}^{-1}|I|^{-1} \cdot \mathcal{K}_{0} \leq \epsilon,
$$

provided that $r N_{0}^{-1}|I|^{-1} \cdot \mathcal{K}_{0}<\epsilon$. Thus, for all $\left(t^{\prime}, x\right) \in J \times B_{r}$,

$$
\left|n\left(t^{\prime}, x\right)-n(p)\right| \leq 2 \epsilon \text {. }
$$

This concludes the proof of the following.

Proposition 4.6. Under assumptions I1, I2, as well as (5) and (6), the globally hyperbolic region of space-time $\mathcal{M}_{I}$ verifies assumption $\mathbf{C}$. More precisely, for every $\epsilon>0$ there exists a constant $r_{0}$, depending only on the fundamental constants $\rho_{0}, I_{0}, N_{0}, \mathcal{K}_{0}, \mathcal{R}_{0}$, such that every point $p \in \mathcal{M}_{I}$ admits a coordinate neighborhood $I_{p} \times U_{p}$, with each $U_{p}$ containing a geodesic ball $B_{r_{0}}(p)$ of radius $r_{0}$, and a system of transported coordinates $(t, x)$ such that (14), (15) and (16) hold true.

The proof of Theorem 2.12 is now an immediate consequence of Theorem 2.11 and Proposition 4.6 .

\section{Curvature Bounds}

In this section we derive $L^{2}$ bounds for the space-time curvature tensor using our assumptions (5) and (6) on the lapse $n$ and second fundamental form $k$ of the $t$-foliation.

5.1. Basic definitions and inequalities. We start with a quick review of the Bel-Robinson tensor and the corresponding energy inequalities induced by $\mathbf{T}$. The fully symmetric, traceless and divergence free Bel-Robinson tensor is given by

$$
\mathbf{Q}[\mathbf{R}]_{\alpha \beta \gamma \delta}=\mathbf{R}_{\alpha \lambda \gamma \mu} \mathbf{R}_{\beta \delta}^{\lambda \mu}+{ }^{\star} \mathbf{R}_{\alpha \lambda \gamma \mu}{ }^{\star} \mathbf{R}_{\beta \delta}^{\lambda \mu} .
$$

The curvature tensor $\mathbf{R}$ can be decomposed into its electric and magnetic parts $E, H$ as follows:

$$
E(X, Y)=\mathbf{g}(\mathbf{R}(X, \mathbf{T}) \mathbf{T}, Y), \quad H(X, Y)=\mathbf{g}\left({ }^{\star} \mathbf{R}(X, \mathbf{T}) \mathbf{T}, Y\right)
$$

with ${ }^{\star} \mathbf{R}$ the Hodge dual of $\mathbf{R}$. One can easily check that $E$ and $H$ are tangent, traceless 2-tensors to $\Sigma_{t}$ and that $|\mathbf{R}|^{2}=|E|^{2}+|H|^{2}$. We easily check the formulas relative to an orthonormal frame $e_{0}=T, e_{1}, e_{2}, e_{3}$ :

$$
\begin{aligned}
& \mathbf{R}_{a b c 0}=-\epsilon_{a b s} H_{s c}, \quad{ }^{\star} \mathbf{R}_{a b c 0}=\epsilon_{a b s} E_{s c}, \\
& \mathbf{R}_{a b c d}=\epsilon_{a b s} \in \epsilon_{c d t} E_{s t}, \quad{ }^{\star} \mathbf{R}_{a b c d}=-\epsilon_{a b s} \in_{c d t} H_{s t} .
\end{aligned}
$$

Observe that

$$
|\mathbf{Q}| \leq 4\left(|E|^{2}+|H|^{2}\right)
$$


and

$$
\mathbf{Q}_{0000}=|E|^{2}+|H|^{2} \text {. }
$$

Let $\mathbf{P}_{\alpha}=\mathbf{Q}[\mathbf{R}]_{\alpha \beta \gamma \delta} \mathbf{T}^{\beta} \mathbf{T}^{\gamma} \mathbf{T}^{\delta}$. By a straightforward calculation,

$$
\mathbf{D}^{\alpha} \mathbf{P}_{\alpha}=\frac{3}{2}{ }^{(\mathbf{T})} \pi^{\alpha \beta} \mathbf{Q}_{\alpha \beta \gamma \delta} \mathbf{T}^{\gamma} \mathbf{T}^{\delta}
$$

Therefore, integrating in a slab $\mathcal{M}_{J}=\bigcup_{t \in J} \Sigma_{t}, J=\left[t_{0}, t\right] \subset I$, we derive the following:

$$
\int_{\Sigma_{t}} \mathbf{Q}_{0000}=\int_{\Sigma_{0}} \mathbf{Q}_{0000}+\frac{3}{2} \int_{t_{0}}^{t} \int_{\Sigma_{t^{\prime}}} n^{(\mathbf{T})} \pi^{\alpha \beta} \mathbf{Q}_{\alpha \beta 00} d v_{g}
$$

with $d v_{g}$ denoting the volume element on $\Sigma_{t}$. Now,

$$
\begin{aligned}
\left|\int_{t_{0}}^{t} \int_{\Sigma_{t^{\prime}}}{ }^{(\mathbf{T})} \pi^{\alpha \beta} \mathbf{Q}_{\alpha \beta 00} d v_{g}\right| & \lesssim N_{0} \int_{t_{0}}^{t} \int_{\Sigma_{t^{\prime}}}{ }^{(\mathbf{T})} \pi \mid\left(|E|^{2}+|H|^{2}\right) d v_{g} \\
& \lesssim N_{0} \int_{t_{0}}^{t}\left\|{ }^{(\mathbf{T})} \pi\left(t^{\prime}\right)\right\|_{L^{\infty}}\left(\left\|E\left(t^{\prime}\right)\right\|_{L^{2}}+\left\|H\left(t^{\prime}\right)\right\|_{L^{2}}\right) d t^{\prime} .
\end{aligned}
$$

Thus, if we denote

$$
\mathcal{Q}(t)=\int_{\Sigma_{t}} \mathbf{Q}_{0000}=\int_{\Sigma_{t}}\left(|E|^{2}+|H|^{2}\right) d v_{g}
$$

we deduce

$$
\mathcal{Q}(t)-\mathcal{Q}\left(t_{0}\right) \lesssim N_{0} \int_{t_{0}}^{t}\left\|\pi\left(t^{\prime}\right)\right\|_{L^{\infty}} \mathcal{Q}\left(t^{\prime}\right) d t^{\prime}
$$

and by Gronwall,

$$
\mathcal{Q}(t) \lesssim \mathcal{Q}\left(t_{0}\right) \exp \left(\int_{t_{0}}^{t} N_{0}\left\|{ }^{(\mathbf{T})} \pi\left(t^{\prime}\right)\right\|_{L^{\infty}} d t^{\prime}\right) .
$$

Thus, in view of (6),

$$
\mathcal{Q}(t) \lesssim \mathcal{Q}\left(t_{0}\right) \exp \left(N_{0} \mathcal{K}_{0}\right) .
$$

We have just proved the following.

Proposition 5.2. Assume that the assumptions (5) and (6) are true. There exists a constant $C=C\left(N_{0}, \mathcal{K}_{0}\right)$ such that, for any $t \in I$,

$$
\|\mathbf{R}(t)\|_{L^{2}} \leq C\left\|\mathbf{R}\left(t_{0}\right)\right\|_{L^{2}}=C \mathcal{R}_{0} .
$$

Instead of integrating (42) in the slab $\mathcal{M}_{J}$ we will now integrate it in the region $\mathcal{D}_{J}^{-}(P)=\mathcal{J}^{-}(p) \cap \mathcal{M}_{J}$ whose boundary consists of the null part $\mathcal{N}^{-}(p)$ and spacelike part $D_{0}(p)=\mathcal{J}^{-}(p) \cap \Sigma_{0}$. We recall that $\mathcal{N}^{-}(p)$ is a Lipschitz manifold and that the set of its terminal points $\mathcal{T}^{-}(p)$ has measure zero relative to $d A_{\mathcal{N}^{-}(p)}$.

Let $\left(\mathbf{P}^{*}\right)_{a \beta \gamma}=\epsilon_{\alpha \beta \gamma \mu} \quad \mathbf{P}^{\mu}$ and the associated differential form, ${ }^{*} \mathbf{P}=$ $\left({ }^{*} \mathbf{P}\right)_{\alpha \beta \gamma} d x^{\beta} d x^{\gamma} d x^{\delta}$. We can rewrite equation (42) in the form $d^{*} \mathbf{P}=-{ }^{*} \mathbf{F}$, with $\left({ }^{*} \mathbf{F}\right)_{\alpha \beta \gamma \delta}=\epsilon_{\alpha \beta \gamma \delta} \mathbf{F}$, and

$$
\mathbf{F}=\frac{3}{2}{ }^{(\mathbf{T})} \pi^{\alpha \beta} \mathbf{Q}_{\alpha \beta \gamma \delta} \mathbf{T}^{\gamma} \mathbf{T}^{\delta} .
$$


Integrating the last expression in the space-time region $\mathcal{D}_{I}^{-}(p)=\mathcal{J}^{-}(p) \cap \mathcal{M}_{J}$, with $J=\left[t_{0}, t\right], p \in \mathcal{M}_{J}$, and applying Stokes theorem, we derive

$$
\int_{\mathcal{D}_{J}^{-}(p)}{ }^{\star} \mathbf{F}=-\int_{\mathcal{D}_{J}^{-}(P)}{ }^{\star} \mathbf{P}=\mathcal{F}_{p}\left(\mathcal{N}^{-}(p) \cap \mathcal{M}_{J}\right)-\operatorname{En}\left(D_{0}(p)\right),
$$

where

$$
\begin{aligned}
\operatorname{En}\left(D_{0}(p)\right) & =-\int_{D_{0}(p)}{ }^{\star} \mathbf{P}=\int_{D_{0}(p)} \mathbf{Q}(\mathbf{T}, \mathbf{T}, \mathbf{T}, \mathbf{T}) d v_{g}, \\
\mathcal{F}_{p}\left(\mathcal{N}^{-}(p) \cap \mathcal{M}_{J}\right) & =-\int_{\mathcal{N}^{-}(p) \cap \mathcal{M}_{J}}{ }^{\star} \mathbf{P} .
\end{aligned}
$$

The energy integral (46) through $D_{0}(p) \subset \Sigma_{0}$ can clearly be bounded by $\left\|\mathbf{R}\left(t_{0}\right)\right\|_{L^{2}}$. Moreover, in view of Proposition 5.2 the integral $\int_{\mathcal{D}_{J}^{-}(p)}{ }^{\star} \mathbf{F}$ can be bounded by $C\left(\mathcal{K}_{0}\right) \cdot \mathcal{R}_{0}$. Therefore,

$$
\mathcal{F}_{p}\left(\mathcal{N}^{-}(p) \cap \mathcal{M}_{J}\right) \lesssim C\left(\mathcal{K}_{0}\right) \cdot \mathcal{R}_{0}
$$

We recall that the null boundary $\mathcal{N}^{-}(p)$ is a Lipschitz manifold. This means that every point $p \in \mathcal{N}^{-1}(p)$ has a local coordinate chart $U_{p}$ together with local coordinate $x^{\alpha}=x^{\alpha}\left(\tau, \omega^{1}, \omega^{2}\right)$ which are Lipschitz continuous. The coordinates are such that for all fixed $20 \omega=\left(\omega^{1}, \omega^{2}\right)$ the curves $\tau \rightarrow x^{\alpha}(\tau, \omega)$ are null, and for any fixed value $\tau$ the 2 dimensional surfaces $S_{\tau}$, given by $x^{\alpha}=x^{a}(\tau, \omega)$, are space-like. In particular there is a well defined null normal $\frac{d x^{\alpha}}{d \tau}=l^{\alpha}$ at all points of $U_{p}$ with the possible exception of a set of measure zero. Moreover we can choose our coordinate charts such that at each point where the normal $l$ is defined we have $\mathbf{g}(l, \mathbf{T})>0$, i.e. $l$ is past oriented. Observe that on such a coordinate chart $U$ we have

$$
\int_{U}{ }^{\star} \mathbf{P}=\int_{U}{ }^{\star} \mathbf{P}_{\alpha \beta \gamma} d x^{\alpha} d x^{\beta} d x^{\gamma}=\int_{U} \mathbf{g}(\mathbf{P}, l) d \tau d A_{\tau}=\int_{U} \mathbf{Q}(\mathbf{T}, \mathbf{T}, \mathbf{T}, l) d \tau d A_{\tau},
$$

with $d A_{\tau}$ the volume element of the space-like surfaces $S_{\tau}$. Since $\mathbf{T}$ is future timelike and $l$ is null past directed we have $\mathbf{Q}(\mathbf{T}, \mathbf{T}, \mathbf{T}, l)<0$. Consequently, for every coordinate chart $U \subset \mathcal{N}^{-}(p), \mathcal{F}_{p}^{-}(U) \geq 0$, where

$$
\mathcal{F}_{p}^{-}(U)=-\int_{U}{ }^{\star} \mathbf{P}
$$

Using a partition of unity it follows that $\mathcal{F}_{p}(U) \geq 0$ for any $U \subset \mathcal{N}^{-}(p)$ and therefore $\mathcal{F}_{p}^{-}\left(U_{1}\right) \leq \mathcal{F}_{p}^{-}\left(U_{2}\right)$ whenever $U_{1} \subset U_{2} \subset \mathcal{N}^{-}(p)$. We can thus identify $\mathcal{F}_{p}(U)$ as the flux of curvature through $U \subset \mathcal{N}^{-}(p)$.

Therefore we have the following:

Proposition 5.3. Under assumptions (5), (6) and (8) the flux of curvature in $\mathcal{M}_{I} \cap \mathcal{N}^{-}(p), \mathcal{F}_{p}^{-}\left(\mathcal{M}_{I}\right)=\mathcal{F}_{p}^{-}\left(\mathcal{M}_{I} \cap \mathcal{N}^{-}(p)\right)$, can be bounded by a uniform constant independent of $p$. More precisely,

$$
\mathcal{F}_{p}\left(\mathcal{M}_{I}\right) \leq C\left(N_{0}, \mathcal{K}_{0}\right) \cdot \mathcal{R}_{0} .
$$

20 The statements here are understood to be true with the possible exception of a set of measure zero relative to the measure $d A_{\mathcal{N}^{-}(p)}$ along $\mathcal{N}^{-}(p)$ introduced just before Definition 2.4 


\section{REFERENCES}

[A1] M. Anderson, Regularity for Lorentz metrics under curvature bounds, J. Math. Phys. 44 (2003), 2994-3012. MR1982778 (2004c:53104)

[A2] M. Anderson, Cheeger-Gromov theory and applications to General Relativity, in: The Einstein Equations and the Large Scale Behavior of Gravitational Fields (Cargese 2002), Ed. P.T. Chruściel and H. Friedrich, Birkauser, Basel (2004), 347-377. MR2098921 (2005f:53055)

[A3] M. Anderson, On long-time evolution in general relativity and geometrization of 3manifolds, Comm. Math. Phys. 222 (2001), 533-567. MR1888088 (2003d:53113)

[AC] M. Anderson, J. Cheeger, Diffeomorphism finiteness for manifolds with Ricci curvature and $L^{\frac{n}{2}}$ curvature bounded, GAFA 1 (1991), 231-251. MR1118730(92h:53052)

[Br] Y. Choquét-Bruhat, Theoreme d'Existence pour certains systemes d'equations aux derivees partielles nonlineaires, Acta Math. 88 (1952), 141-225. MR0053338 (14:756g)

[Ch] J. Cheeger, Finiteness theorems for Riemannian manifolds, Am. J. Math. 92 (1970), 61-75. MR0263092 (41:7697)

[C-K] D. Christodoulou, S. Klainerman, The global nonlinear stability of the Minkowski space, Princeton Math. Series 41, 1993. MR.1316662 (95k:83006)

[Fried] H.G. Friedlander, The Wave Equation on a Curved Space-time, Cambridge University Press, 1976. MR0460898 (57:889)

[G] G. Galloway, Maximum principles for null hypersurfaces and null splitting theorems, Ann. Henri Poincare 1 (2000), 543-567. MR1777311 (2002b:53052)

[HE] S.W. Hawking, G.F.R. Ellis, The Large Scale Structure of Space-time, Cambridge University Press, Cambridge, 1973. MR0424186 (54:12154)

[HKM] T.J.R. Hughes, T. Kato and J.E. Marsden, Well-posed quasi-linear second-order hyperbolic systems with applications to nonlinear elastodynamics and general relativity, Arch. Rational Mech. Anal. 63 (1977), 273-394. MR0420024 (54:8041)

[KR1] S. Klainerman and I. Rodnianski, Causal geometry of Einstein-Vacuum spacetimes with finite curvature flux Inventiones Math. 2005, vol. 159, No. 3, 437-529. MR2125732 (2006e:58042)

[KR2] S. Klainerman and I. Rodnianski, A geometric approach to Littlewood-Paley theory, GAFA 16 (2006), 126-163. MR.2221254 (2007e:58046)

[KR3] S. Klainerman and I. Rodnianski, Sharp trace theorems for null hypersurfaces on Einstein metrics with finite curvature flux, GAFA 16 (2006), 164-229. MR2221255 (2007e:58047)

[KR4] S. Klainerman and I. Rodnianski, A Kirchoff-Sobolev parametrix for the wave equation in curved space-time, preprint

[KR5] S. Klainerman and I. Rodnianski, A large data break-down criterion in General Relativity, submitted to J. Amer. Math. Soc.

[PSW] P. Petersen, S.D. Steingold, G. Wei, Comparison geometry with integral curvature bounds, GAFA 7 (1997), 1011-1030. MR 1487752 (99c:53022)

[Pe] P. Peterson, Convergence theorems in Riemannian geometry, MSRI publications, volume 30, 1997. MR 1452874 (98k:53049)

[Sob] S. Sobolev, Methodes nouvelle a resoudre le probleme de Cauchy pour les equations lineaires hyperboliques normales, Matematicheskii Sbornik, vol. 1 (43) 1936, 31-79.

[Wang] Q. Wang, Causal geometry of Einstein vacuum space-times. Ph.D. thesis, Princeton University, 2006.

Department of Mathematics, Princeton University, Princeton, New Jersey 08544

E-mail address: seri@math.princeton.edu

Department of Mathematics, Princeton University, Princeton, New Jersey 08544

E-mail address: irod@math.princeton.edu 